\title{
ON THE COMPLETENESS OF NON-SYMMETRICAL UNIFORM CONVERGENCE WITH SOME LINKS TO APPROACH SPACES
}

\author{
DIETER LESEBERG AND ZOHREH VAZIRY
}

\begin{abstract}
The quasitopos b-UFIL of b-uniform filter spaces [16] are an appropriate tool for studying convergence from a higher point of view as demonstrated in recent papers by the above mentioned authors. In addition BORN, the category of bornological spaces and bounded maps, can be integrated as bicoreflective subcategory of b-UFIL. As already shown symmetric b-uniform filter spaces have "Cauchy completions" which generalize some important ones as for example those which were considered by Wyler, Preuss, Czászár and Hausdorff, respectively. In the present paper we will construct a completion, called ultracompletion, for a suitable not necessarily symmetric b-uniform filter space and compare this one with a construct presented for quasi-uniform spaces by Carlson and Hicks in the past. Furthermore, among others, we get the result that every quasiuniform limit space in the sense of Behling has an ultracompletion. At the end of this article, we consider some important links to generalized approach spaces, those which were introduced by Lowen. So it is shown that b-topological closure operators can be completely described by so-called approach-bornologies, which represent a common generalization of both approach spaces and bornological spaces, respectively. Thus, as interesting corollary we obtain the result that APB the category of approach-bornological spaces and contracted maps intersects b-URING, the full subcategory of b-UFIL, whose objects have ultracompletions.
\end{abstract}

\section{INTRODUCTION}

In our last paper "The Cauchy-completion of a symmetric b-uniform filter space" we announce the question whether there exist completions for given not necessarily symmetric b-uniform filter spaces. Here, a b-uniform filter space is a triple consisting of a non-empty subset $\mathcal{B}^{X} \subset \underline{P} X$ and a non-empty subset

$$
\mu \subset F I L(X \times X):=\{\mathcal{U}: \mathcal{U} \text { is filter on } X \times X\}
$$

such that the following conditions hold

(buf b $_{1} B_{1} \subset B \in \mathcal{B}^{X}$ implies $B_{1} \in \mathcal{B}^{X}$;

(buf $_{2}$ ) $x \in X$ implies $\{x\} \in \mathcal{B}^{X}$;

(buf $\left._{3}\right) B \in \mathcal{B}^{X} \backslash\{\emptyset\}$ implies $\dot{B} \times \dot{B} \in \mu$, where $\dot{B}:=\{A \subset X: A \supset B\}$;

(buf bu $_{4} \mathcal{U} \in$ and $\mathcal{U} \subset \mathcal{U}_{1} \in F I L(X \times X)$ imply $\mathcal{U}_{1} \in \mu$.

$M S C$ (2010): primary 54D35, 54A05, 54A20; secondary 54B30, 54E15.

Keywords: b-uniform filter space, b-filter space, bornological space, ultracompletion, preCauchy filter, set-convergence, approach space, preuniform convergence space, quasi-uniform space, bounded set, bounded topology. 
In general, for filters $\mathcal{F}$ and $\mathcal{G}$, their cross product is defined by $\mathcal{F} \times \mathcal{G}:=\{R \subset$ $X \times X: \exists F \in \mathcal{F} \exists G \in \mathcal{G}$ s.t. $R \supset F \times G\}$.

For b-uniform filter spaces $\left(X, \mathcal{B}^{X}, \mu_{X}\right),\left(Y, \mathcal{B}^{Y}, \mu_{Y}\right)$ a function $f: X \longrightarrow Y$ is called $b$-uniformly continuous map, provided that the following conditions are satisfied,

(buc 1 ) $B \in \mathcal{B}^{X}$ implies $f[B] \in \mathcal{B}^{Y}$;

$\left(\right.$ buc $\left._{2}\right) \mathcal{U} \in \mu_{X}$ implies $(f \times f)(\mathcal{U}) \in \mu_{Y}$.

By b-UFIL we denote the category of b-uniform filter spaces and b-uniformly continuous maps.

In this context we point out that $\mathcal{B}^{X}$ defines a $\underline{B}$-set in the sense of Wyler [25]. On the other hand if $\mathcal{B}^{X}$ is discrete, meaning that $\mathcal{B}^{X}=\mathcal{D}^{X}:=\{\emptyset\} \cup\{\{x\}$ : $x \in X\}$ holds, then b-uniform filter spaces and preuniform convergence spaces in the sense of Preuss [22] are essentially the same (up to isomorphism); meaning that DISb-UFIL the full subcategory of b-UFIL, whose objects are discrete is isomorphic to PUCONV, the category of preuniform convergence spaces and uniformly continuous maps.

Furthermore the quasi-topos BOUND of bound spaces and bounded maps [23] can also be fully embedded into b-UFIL.

Here, a bound space is a pair $\left(X, \mathcal{B}^{X}\right)$, where $\mathcal{B}^{X}$ denotes a B-set, and the morphisms between them are the bounded maps, compare with $\left(\right.$ buc $\left._{1}\right)$. Then, for an arbitrary $\underline{B}$-set $\mathcal{B}^{X}$ we consider the pair $\left(\mathcal{B}^{X}, \mu_{b}\right)$, where $\mu_{b}$ is defined by setting

$$
\mu_{b}:=\left\{\mathcal{U} \in F I L(X \times X): \exists B \in \mathcal{B}^{X} \text { s.t. } \dot{B} \times \dot{B} \subset \mathcal{U}\right\}
$$

Conversely, for a bounded b-uniform filter space $\left(X, \mathcal{B}^{X}, \eta\right)$, meaning that $\left(\mathcal{B}^{X}, \eta\right)$ is satisfying the following condition,

(B) $\mathcal{V} \in \eta$ implies the existence of an $B \in \mathcal{B}^{X}$ with $B \times B \in \mathcal{V}$,

the so defined assignments deliver an isomorphism between the category BOUND and B-UFIL, the full subcategory of b-UFIL whose objects are bounded. Furthermore B-UFIL is bicoreflective in b-UFIL. In fact let $\left(X, \mathcal{B}^{X}, \mu\right)$ be a buniform filter space, then by setting $\mu^{B}:=\left\{\mathcal{U} \in \mu: \exists B \in \mathcal{B}^{X} B \times B \in \mathcal{U}\right\}$ we obtain a bounded b-uniform filter space such that $1_{X}:\left(X, \mathcal{B}^{X}, \mu^{B}\right) \longrightarrow\left(X, \mathcal{B}^{X}, \mu\right)$ is the bicoreflection of $\left(X, \mathcal{B}^{X}, \mu\right)$ with respect to B-UFIL. Now, in addition let us call a b-uniform filter space $\left(X, \mathcal{B}^{X}, \mu\right)$ bornological provided that $\mathcal{B}^{X}$ forms a bornology, meaning that $\mathcal{B}^{X}$ in addition satisfies $B_{1}, B_{2} \in \mathcal{B}^{X}$ imply $B_{1} \cup B_{2} \in$ $\mathcal{B}^{X}$. By BONb-UFIL we denote the full subcategory of b-UFIL, whose objects are bornological. Then, the category BORN of bornological spaces and bounded maps, [9] can be regarded as full subcategory of the intersection of BONb-UFIL and B-UFIL, respectively. Finally we still mention the concept of final b-uniform filter spaces, regarded as full subcategory of b-UFIL, denoted by FINb-UFIL and whose objects are final, meaning that the corresponding $\underline{B}$-sets are satisfying the following condition,

(f) $B \in \mathcal{B}^{X}$ implies $B$ is finite.

Here we note that every finite b-uniform filter space is final, and furthermore we state that each discrete b-uniform filter space is final, too. 
Thus final b-uniform filter spaces represent roughly spoken a common generalization of uniform convergence and the bornologies of finite sets. Later (see up to 5.11) we will see which important role they are playing in the context of ultracompleteness.

Finally, we infer that FINb-UFIL is bicoreflective in b-UFIL. To these facts, we note that in our view filters may contain the empty set. This is of importance, too, if one considers set-convergence spaces in the sense of Wyler [25].

A set-convergence is a pair $\left(\mathcal{B}^{X}, q\right)$, where $\mathcal{B}^{X}$ is $\underline{B}$-set and $q \subset \mathcal{B}^{X} \times F I L(X)$, such that the following conditions are satisfied,

$\left(\mathrm{SC}_{1}\right) B \in \mathcal{B}^{X}$ implies $(B, \dot{B}) \in q$;

$\left(\mathrm{SC}_{2}\right)(\emptyset, \mathcal{F}) \in q$ implies $\mathcal{F}=\underline{P X}$

$\left(\mathrm{SC}_{3}\right)(B, \mathcal{F}) \in q$ and $\mathcal{F} \subset \mathcal{F}_{1} \in F I L(X)$ imply $\left(B, \mathcal{F}_{1}\right) \in q$.

Then, the triple $\left(X, \mathcal{B}^{X}, q\right)$ is called a set-convergence space. Often we write $\mathcal{F} q B$ iff $(B, \mathcal{F}) \in q$ is valid.

A function $f: X \rightarrow Y$ between set-convergence spaces $\left(X, \mathcal{B}^{X}, q_{X}\right),\left(Y, \mathcal{B}^{Y}, q_{Y}\right)$ is called $b$-continuous provided that $f$ is bounded, and in addition, $f$ transfers convergent filters. By SETCONV we are denoting the corresponding category. An important subcategory of SETCONV is the bireflective full subcategory ROSETCONV, whose objects are reordered. Here, a set-convergence $\left(\mathcal{B}^{X}, q\right)$ is called reordered, and the triple $\left(X, \mathcal{B}^{X}, q\right)$ a reordered set-convergence space, provided it satisfies the following conditions,

$(\mathrm{RO}) \emptyset \neq B_{1} \subset B \in \mathcal{B}^{X}$ and $\mathcal{F} q B$ imply $\mathcal{F} q B_{1}$.

Here, we should mention that now classical point-convergence spaces, such as KENT-convergence spaces, limit spaces, pseudo-topological spaces, pre-topological spaces or topological spaces as well can be regarded as special reordered setconvergence spaces by restricting $\mathcal{B}^{X}$ to $\mathcal{D}^{X}[6]$ (see Section 3 ).

Note also that RO-SETCONV can be fully embedded into b-UFIL as follows: For reordered set-convergence space $\left(X, \mathcal{B}^{X}, q\right)$ we consider the triple $\left(X, \mathcal{B}^{X}, \mu_{q}\right)$, where $\mu_{q}$ is defined by setting:

$$
\begin{aligned}
\mu_{q}:=\left\{\mathcal{U} \in F I L(X \times X): \exists \mathcal{F} \in F I L(X) \exists B \in \mathcal{B}^{X} \backslash\{\emptyset\}\right. \\
\qquad(\mathcal{F} q B \text { and } \dot{B} \times \mathcal{F} \subset \mathcal{U})\} \cup\{\underline{P}(X \times X)\} .
\end{aligned}
$$

Then, $\left(\mathcal{B}^{X}, \mu_{q}\right)$ forms a so-called b-uniform convergence, and the triple $\left(X, \mathcal{B}^{X}, \mu_{q}\right)$ a $b$-uniform convergence space.

Here, a b-uniform filter space $\left(X, \mathcal{B}^{X}, \eta\right)$ is called b-uniform convergence space, provided that $\left(\mathcal{B}^{X}, \eta\right)$ satisfies the following condition,

(cv) $\mathcal{U} \in \eta$ implies the existence of $\mathcal{F} \in F I L(X)$ and $B \in \mathcal{B}^{X}$ with $\mathcal{U} \supset \dot{B}$ $\times \mathcal{F} \in \eta$.

In this context $\mathcal{F} p_{\eta} B$ is defined by setting:

$$
\begin{array}{lll}
\mathcal{F} p_{\eta} \emptyset & \text { iff } & \mathcal{F}=\underline{P} X \text { and } \\
\mathcal{F} p_{\eta} B & \text { iff } & \dot{B} \times \mathcal{F} \in \eta \text { for every } B \in \mathcal{B}^{X} \backslash\{\emptyset\} .
\end{array}
$$

The so-defined assignments deliver an isomorphism between the categories 
whose objects are the b-uniform convergence spaces. Thus our new developed concept seems to be an appropriate tool for a common study of all former investigated categories.

In giving some intrinsic examples let us consider a quasi-uniform space $(X, \mathcal{U})$, [12] and let $\mathcal{U}^{-1}:=\left\{U^{-1}: U \in \mathcal{U}\right\}$ be the conjugate quasi-uniformity, where $U^{-1}:=\{(y, x):(x, y) \in U\}$. If $\mathcal{S}$ is a non-empty family of subsets of $X$, we may consider the following three convergences on $\underline{P} X$. Let $\left(A_{t}\right)$ be a net of subsets of $X$ and $A \subset X$. We say that the net $\left(A_{t}\right)$ :

$\mathcal{S}^{-}$-converges to $A$, and we write $A_{t} \stackrel{\mathcal{S}^{-}}{\longrightarrow} A$ provided for each $S \in \mathcal{S}$ and $U \in \mathcal{U}$ there exists $t_{0}$ such that $A \cap S \subset U^{-1}\left(A_{t}\right)$ for every $t \geq t_{0}$;

$\mathcal{S}^{+}$-converges to $A$, and we write $A_{t} \stackrel{\mathcal{S}^{+}}{\longrightarrow} A$, provided for each $S \in \mathcal{S}$ and $U \in \mathcal{U}$ there exists $t_{0}$ such that $A_{t} \cap S \subset U(A)$ for every $t \geq t_{0}$;

$\widehat{\mathcal{S}}$-converges to $A$ provided it $\mathcal{S}^{-}$-converges to $A$ and it $\mathcal{S}^{+}$-converges to $A$, where $U(A):=\{y \in X:(x, y) \in U$ for some $x \in A\}, U^{-1}(A):=\{y \in X:$ $(x, y) \in U^{-1}$ for some $\left.x \in A\right\}$.

Note that if $X$ is a metric space and $\mathcal{S}=\{X\}$ then the $\widehat{\mathcal{S}}$-converges on the space of closed bounded subsets of $X$ is simply the $H$-convergence, i.e. the convergence in the Hausdorff metric. The just presented ideas are going back to A. Lechicki, S. Levi and A. Spakowski [13] where in their work Bornological converges one also can find additional examples and corresponding references, see also [24].

Now, to tackle our problem we have to alter the definition of a $\mu$-Cauchy filter [17] so that, in the symmetric case, they coincide.

\section{BASIC NOTIONS}

Definition 2.1. Let $\left(X, \mathcal{B}^{X}, \mu\right)$ be a b-uniform filter space, $\mathcal{C} \in F I L(X) \backslash\{\underline{P} X\}$ is called pre-Cauchy filter (shortly pre-Chy filter) in $\left(\mathcal{B}^{X}, \mu\right)$, provided $\mathcal{C}$ satisfies the following condition,

(pChy) $\exists \mathcal{U} \in \mu$ s.t. $\forall R \in \mathcal{U} \exists B \in \mathcal{B}^{X} \backslash\{\emptyset\}$ s.t. $R(B) \in \mathcal{C}$.

Here $R(B):=\{z \in X: \exists x \in B$ s.t. $(x, z) \in R\}$.

Remark 2.2. Here we should note that in the discrete case for a symmetric b-uniform net space $\left(X, \mathcal{B}^{X}, \mu\right)$ and a filter $\mathcal{C} \in F I L(X) \backslash\{\underline{P} X\}$ the following statements are equivalent:

(i) $\mathcal{C}$ is pre-Chy filter in $\left(\mathcal{B}^{X}, \mu\right)$;

(ii) $\mathcal{C}$ is $\mu$-Chy filter [17].

Here, $\mathcal{C} \in F I L(X) \backslash\{\underline{P} X\}$ is called $\mu$-Chy filter iff $\mathcal{C} \times \mathcal{C} \in \mu$ is valid. Thus, if returning to the discrete case $\mathcal{C}$ defines a Cauchy-filter as usual on the associated uniform limit space, [22]. Moreover, we note that roughly spoken the b-uniformly continuous image of a pre-Chy filter is a pre-Chy filter again.

Definition 2.3. Let $\left(X, \mathcal{B}^{X}, \mu\right)$ be a b-uniform filter space. Then, $\mathcal{F} \in F I L(X)$ is called setconvergent in $\left(\mathcal{B}^{X}, \mu\right)$ iff there exists $B \in \mathcal{B}^{X} \backslash\{\emptyset\}$ s.t. $\dot{B} \times \mathcal{F} \in \mu$.

Remark 2.4. By the definition used in Introduction, this can be expressed by the fact that $\mathcal{F} p_{\mu} B$ is valid for some $B \in \mathcal{B}^{X} \backslash\{\emptyset\}$. 
Now it is clear that every setconvergent filter $\mathcal{F} \in F I L(X) \backslash\{\underline{P} X\}$ in $\left(\mathcal{B}^{X}, \mu\right)$ is a pre-Chy filter. In this context we also note that for an reordered set-convergence space $\left(X, \mathcal{B}^{X}, q\right)$ and a filter $\mathcal{F} \in F I L(X)$ the following statements are equivalent:

(i) $\mathcal{F} q B$ for some $B \in \mathcal{B}^{X} \backslash\{\emptyset\}$;

(ii) $\mathcal{F} q\{x\}$ for some $x \in X$.

Definition 2.5. A b-uniform filter space $\left(X, \mathcal{B}^{X}, \mu\right)$ is called ultracomplete, provided that every pre-Chy filter in $\left(\mathcal{B}^{X}, \mu\right)$ is setconvergent.

Example 2.6. Every non-empty bornological bounded b-uniform filter space $\left(X, \mathcal{B}^{X}, \mu\right)$ is ultracomplete.

Proof. Let $\mathcal{C} \in F I L(X) \backslash\{\underline{P} X\}$ be a pre-Chy filter in $\left(\mathcal{B}^{X}, \mu\right)$, hence we can find $\mathcal{U} \in \mu$ with the corresponding property. But by the hypothesis $\left(\mathcal{B}^{X}, \mu\right)$ is bounded, thus $B \times B \in \mathcal{U}$ for some $B \in \mathcal{B}^{X}$. Then, we can choose $D \in \mathcal{B}^{X} \backslash\{\emptyset\}$ s.t. $(B \times B)(D) \in \mathcal{C}$ by applying the pre-Cauchy property, hence $D \cup B \in \mathcal{B}^{X} \backslash\{\emptyset\}$, since $\mathcal{B}^{X}$ is bornology. Consequently, $(D \dot{\cup} B) \times(D \dot{\cup} B) \in \mu$ follows. It remains to prove $(D \dot{\cup} B) \subset \mathcal{C}$, because then $\mathcal{C} q_{\mu} D \cup B$ is valid.

$A \in(D \cup B)$ implies $A \supset D \cup B$. We will show that the inclusion $D \cup B \subset$ $(B \times B)(D)$ holds. $z \in(B \times B)(D)$ implies the existence of $x \in D$ such that $(x, z) \in B \times B$, and $z \in D \cup B$ follows, proving the claim.

Example 2.7. Every non-empty b-uniform convergence space $\left(X, \mathcal{B}^{X}, \mu\right)$ is ultracomplete.

Proof. Let $\mathcal{C} \in F I L(X) \backslash\{\underline{P} X\}$ be a pre-Chy filter in $\left(\mathcal{B}^{X}, \mu\right)$, hence we can find an $\mathcal{U} \in \mu$ with the corresponding property. Since $\left(X, \mathcal{B}^{X}, \mu\right)$ is b-uniform convergence space, there exists $B \in \mathcal{B}^{X}$ and a filter $\mathcal{F} \in F I L(X)$ such that $\mathcal{U} \supset \dot{B} \times \mathcal{F} \in \mu$ are valid. $B$ is not empty and $\mathcal{F}$ is subfilter of $\mathcal{C}$ and thus $\mathcal{F} \neq \underline{P} X$. Because $F \in \mathcal{F}$ implies $B \times F \in \mathcal{U}$, hence we can find $D \in \mathcal{B}^{X} \backslash\{\emptyset\}$ with $(B \times F)(D) \in \mathcal{C}$. Since $(B \times F)(D) \subset F$ holds, $F \in \mathcal{C}$ follows, and consequently $\dot{B} \times \mathcal{C} \in \mu$ results, which shows $\mathcal{C} q_{\mu} B$.

Now, we introduce two further important notions which are closed to the former presented concept.

Definition 2.8. A b-uniform filter space $\left(X, \mathcal{B}^{X}, \mu\right)$ is called

(i) ultracompact provided that each ultrafilter $\mathcal{F} \in F I L(X) \backslash\{\underline{P} X\}$ is setconvergent in $\left(\mathcal{B}^{X}, \mu\right)$;

(ii) ultrabounded provided that each ultrafilter $\mathcal{F} \in F I L(X) \backslash\{\underline{P} X\}$ is a preChy filter in $\left(\mathcal{B}^{X}, \mu\right)$.

Remark 2.9. Evidently, each non-empty finite b-uniform filter space is ultracompact. Moreover, every ultracompact b-uniform filter space is ultrabounded, and in addition we get that every ultrabounded and ultracomplete b-uniform filter space is ultracompact again.

Furthermore we note that, supposing a symmetric b-uniform net space $\left(X, \mathcal{B}^{X}, \mu\right)$, then $\mathcal{F} \in F I L(X)$ is setconvergent in $\left(\mathcal{B}^{X}, \mu\right)$ iff $\mathcal{F} q_{\tau_{\mu}} B$ is valid for some $B \in \mathcal{B}^{X} \backslash\{\emptyset\}$. 
Here $\mathcal{F} q_{\tau_{\mu}} B$ iff $\mathcal{F} \cap \dot{B} \in \tau_{\mu}$ [15]. Thus we get that for a symmetric b-uniform net space the terms compactness and ultracompactness are essentially the same. Then, finally, if considering the discrete case we obtain the fundamental result that a symmetric b-uniform net space is ultracompact iff it is ultrabounded and ultracomplete. Now taking all these facts into account we resume that the new terms introduced generalize the older ones of compactness, precompactness and completeness, respectively in a rather natural way.

\section{More ABout SET-COnvergence}

Returning to the concept of reordered set-convergence we should explain how it plays an important role for further studies of point-convergences, not only restricted to the discrete case.

Now, if one considers point-convergence on arbitrary B-sets, such as the set $\mathcal{E}^{X}$ of all finite subsets or the set $\tau^{X}$ of all totally bounded subsets or the set $\mathcal{C}^{X}$ of all compact subsets of a set $X$ we extend the basics to the following one, i.e.

Definition 3.1. We call a reordered set-convergence $\left(\mathcal{B}^{X}, q\right)$ pointset- convergence (on $X$ ), and the triple $\left(X, \mathcal{B}^{X}, q\right)$ pointset - convergence space, provided that the following condition is satisfied, i.e.

(pset) $\mathcal{F} \in F I L(X), B \in \mathcal{B}^{X} \backslash\{\emptyset\}$ and $\mathcal{F} q\{x\} \forall x \in B$ imply $\mathcal{F} q B$.

Remark 3.2. Here we point out, that each discrete set-convergence space is a pointset-convergence space. Convergence in the usual sense like limit spaces or pretopological spaces, respectively are being involved [6]. Consequently, all possible point-convergences on any $\underline{B}$-sets can be now subsumed under the concept of pointset-convergence spaces. If we denote by P-SETCONV the corresponding full subcategory of RO-SETCONV, then we claim that P-SETCONV is bireflective in RO-SETCONV.

In fact for a reordered set-convergence space $\left(X, \mathcal{B}^{X}, q\right)$ we put for $\mathcal{F} \in F I L(X)$ and $B \in \mathcal{B}^{X} \backslash\{\emptyset\} \mathcal{F} \dot{q} \quad B$ iff $\mathcal{F} q\{x\} \forall x \in B$, and $\mathcal{F} \dot{q} \emptyset$ iff $\mathcal{F}=\underline{P} X$. Then, $\left(X, \mathcal{B}^{X}, \stackrel{\dot{q}}{)}\right)$ is a pointset-convergence space such that the demand for the bireflection is satisfied.

Another point of view is considering the set-convergence $\left(\mathcal{B}^{X}, q_{\mu}\right)$ being induced by a given generated b-uniform filter space $\left(X, \mathcal{B}^{X}, \mu\right)$. As already seen $[17,22]$ generated b-uniform filter spaces are in one-to-one correspondence with principal preuniform convergence spaces or preuniform spaces or diagonal filters in the sense of Weil [1] by assuming the discrete case. So we are coming quite naturally to the following definition:

Definition 3.3. A set-convergence $\left(\mathcal{B}^{X}, q\right)$ is called set-surrounding and the triple $\left(X, \mathcal{B}^{X}, q\right)$ set-surrounding space, provided that the following property is valid,

(ss) $B \in \mathcal{B}^{X} \backslash\{\emptyset\}$ implies $\bigcap\{\mathcal{F}: \mathcal{F} q B\} q B$.

Remark 3.4. Let a neighborhood space $\left(X, \mathcal{B}^{X}, \Theta\right)$ be given [23], then the triple $\left(X, \mathcal{B}^{X}, q_{\Theta}\right)$ defines a set-surrounding space, where

$$
\mathcal{F} q_{\Theta} B \text { iff } \mathcal{F} \supset \Theta(B) \forall B \in \mathcal{B}^{X} \text {. }
$$


Lemma 3.5. If $\left(X, \mathcal{B}^{X}, \mu\right)$ is a generated b-uniform filter space, then the underlying set convergence $\left(\mathcal{B}^{X}, q_{\mu}\right)$ is a set-surrounding on $X$ and determines the set-surrounding space $\left(X, \mathcal{B}^{X}, q_{\mu}\right)$.

Proof. Let $B \in \mathcal{B}^{X} \backslash\{\emptyset\}$ and consider all $\mathcal{F} \in F I L(X)$ s.t. $\mathcal{F} q_{\mu} B$ is valid, hence $\dot{B} \times \mathcal{F} \in \mu$ holds for those $\mathcal{F} \in F I L(X)$. It remains to show that the statement $\dot{B} \times \cap\left\{\mathcal{F} \in F I L(X): \mathcal{F} q_{\mu} B\right\} \in \mu$ can be deduced. Therefore, it suffices to show that the inclusion $\cap \mu \subset \dot{B} \times \cap\left\{\mathcal{F} \in F I L(X): \mathcal{F} q_{\mu} B\right\}$ holds, since by the hypothesis $\mu$ is generated. So let $R \in \mu$, hence $R \supset B \times F_{\mathcal{F}}$ for some $F_{\mathcal{F}} \in \mathcal{F}$ with $\mathcal{F} q_{\mu} B$. Consequently, $R \supset \cup\left\{B \times F_{\mathcal{F}}: \mathcal{F} q_{\mu} B\right\} \supset B \times \cup\left\{F_{\mathcal{F}}: \mathcal{F} q_{\mu} B\right\}$, because $(x, z) \in B \times \cup\left\{f_{\mathcal{F}}: \mathcal{F} q_{\mu} B\right\}$ implies $x \in B$, and there exists $\mathcal{F}^{\prime} \in F I L(X)$ with $\mathcal{F}^{\prime} q_{\mu} B$ and $z \in F_{\mathcal{F}}^{\prime}$. Consequently, $(x, z) \in B \times F_{\mathcal{F}^{\prime}}$ implies $(x, z) \in$ $\cup\left\{B \times F_{\mathcal{F}}: \mathcal{F} q_{\mu} B\right\}$. But $B \times \cup\left\{F_{\mathcal{F}}: \mathcal{F} q_{\mu} B\right\} \in \dot{B} \times \cap\left\{\mathcal{F} \in F I L(X): \mathcal{F} q_{\mu} B\right\}$, and the claim follows.

Remark 3.6. Here we point out, that in the discrete case $\left(X, \mathcal{B}^{X}, q_{\mu}\right)$ even defines a pretopological space. Then, a related expression aims at so-called closure operators. Indeed, let a b-uniform filter space $\left(X, \mathcal{B}^{X}, \mu\right)$ be given, then we can define the following two closure spaces $\left(X, c l_{\mu}\right)$ and $\left(X, c l^{\mu}\right)$ by setting:

$$
\begin{aligned}
& c l_{\mu}(\emptyset):=\emptyset \text { and } \\
& c l_{\mu}(A):=\{x \in X: \exists \mathcal{F} \in F I L(X)(\dot{x} \times \mathcal{F} \in \mu \text { and } A \in \sec \mathcal{F})\} \forall(\emptyset \neq A) \in \underline{P} X,
\end{aligned}
$$

where $\sec \mathcal{F}:=\{D \subset X: \forall F \in \mathcal{F} F \cap D \neq \emptyset\}$.

Respectively, we put:

$$
\begin{aligned}
& c l^{\mu}(\emptyset):=\emptyset \text { and } \\
& c l^{\mu}(A):=\{x \in X: \exists \mathcal{U} \in \mu \text { s.t. }\{x\} \times A \in \sec \mathcal{U}\}, \forall(\emptyset \neq A) \in \underline{P} X
\end{aligned}
$$

Here, in general we note that $c_{\mu}(A) \subset c l^{\mu}(A)$ is true for every $A \in \underline{P} X$. In the case of $\left(X, \mathcal{B}^{X}, \mu\right)$ being b-uniform convergence space then the equality of both the closures results. The latter closure defined will be used in 5.1 in obtaining that $X$ is dense in $X^{*}$.

\section{B-UNIFORM RING SPACES}

Before coming to the core of this article, we introduce the following important notion.

Definition 4.1. For a b-uniform filter space $\left(X, \mathcal{B}^{X}, \mu\right)$ a pair $\left(\mathcal{A}^{X}, \eta\right)$ with $\emptyset \neq \mathcal{A}^{X} \subset \underline{P} X$ and $\emptyset \neq \eta \in F I L(X \times X)$ is called a base for $\left(\mathcal{B}^{X}, \mu\right)$, provided that the following equations hold, (bas $\left._{1}\right) \mathcal{B}^{X}=\left\{B \subset X: \exists A \in \mathcal{A}^{X}\right.$ s.t. $\left.B \subset A\right\}$ and $\left(\right.$ bas $\left._{2}\right) \mu=\{\mathcal{V} \in F I L(X \times X): \exists \mathcal{U} \in \eta$ s.t. $\mathcal{U} \subset \mathcal{V}\}$ (compare with [1]).

Remark 4.2. Here we should note that a pair $\left(\mathcal{A}^{X}, \eta\right)$ is a base for a b-uniform filter structure on $X$ iff it satisfies the following conditions:

(bsuf $\left._{1}\right) \emptyset \in \mathcal{A}^{X}$

(bsuf $_{2}$ ) $x \in X$ implies $\{x\} \in \mathcal{A}^{X}$; 
(bsuf $) \forall B \in \mathcal{B}^{X} \backslash\{\emptyset\} \exists \mathcal{U} \in \eta$ s.t. $\mathcal{U} \subset \dot{B} \times \dot{B}$.

Lemma 4.3. For b-uniform filter spaces $\left(X, \mathcal{B}^{X}, \mu_{X}\right),\left(Y, \mathcal{B}^{Y}, \mu_{Y}\right)$ let $f: X \longrightarrow$ $Y$ be a map. Let us denote by $\left(\mathcal{A}^{X}, \eta_{X}\right)$ respectively $\left(\mathcal{A}^{Y}, \eta_{Y}\right)$ bases for the corresponding spaces. Then, the following statements are equivalent:

(i) $f:\left(X, \mathcal{B}^{X}, \mu_{X}\right) \longrightarrow\left(Y, \mathcal{B}^{Y}, \mu_{Y}\right)$ is b-uniformly continuous;

(ii) (1) $A \in \mathcal{A}^{X}$ implies $\exists A^{Y} \in \mathcal{A}^{Y}$ s.t. $f[A] \subset A^{Y}$;

(2) $\mathcal{U} \in \mu_{X}$ implies $\exists \mathcal{U}_{Y} \in \eta_{Y}$ s.t. $\mathcal{U}_{Y} \subset(f \times f)(\mathcal{U})$.

Proof. By straightforward executing.

As pointed out by many authors in the past, quasi-uniform spaces, quasiuniform convergence spaces, Cauchy spaces or point-convergence spaces, respectively are also of interest if one considers suitable extensions of the given constructs $[4,5,7$, $8,11,14]$.

Here we note again that all the above mentioned spaces can be simply described by the associated b-uniform filter spaces. Moreover each of them fulfills an additional common property, which we will be now described as follows:

Definition 4.4. A b-uniform filter structure $\left(\mathcal{B}^{X}, \mu\right)$ is called b-uniform ring structure (on $X$ ) and the space $\left(X, \mathcal{B}^{X}, \mu\right)$ b-uniform ring space, provided it satisfies the following condition, i.e.

(rg) $\mathcal{U} \in \mu$ implies $\mathcal{U} \circ \mathcal{U} \in \mu$, where in general for filters $\mathcal{U}, \mathcal{V} \in F I L(X \times X)$, $\mathcal{U} \circ \mathcal{V}:=\{R \subset X \times X: \exists U \in \mathcal{U} \exists V \in \mathcal{V}$ s.t. $R \supset U \circ V\}$.

Examples 4.5. (i) Let $(X, \mathcal{U})$ be a quasi-uniform space. Then, the associated space $\left(X, \mathcal{D}^{X}, \mu_{\mathcal{U}}\right)$ defines a b-uniform ring space, where $\mu_{\mathcal{U}}:=$ $\{\mathcal{V} \in F I L(X \times X): \mathcal{V} \supset \mathcal{U}\}$. Note, that the pair $\left(\mathcal{D}^{X},\{\mathcal{U}\}\right)$ defines a base for $\left(\mathcal{D}^{X}, \mu_{\mathcal{U}}\right)$

(ii) Let $\left(X, J_{X}\right)$ be a quasiuniform convergence space. Then, the associated space $\left(X, \mathcal{D}^{X}, J_{X}\right)$ defines a b-uniform ring space;

(iii) for a b-filter space $\left(X, \mathcal{B}^{X}, \Gamma\right)[11,15,17]$ the space $\left(X, \mathcal{B}^{X}, \mu_{\Gamma}\right)$ defines a b-uniform ring space, where the pair $\left(\mathcal{B}^{X},\{\mathcal{F} \times \mathcal{F}: \mathcal{F} \in \Gamma\}\right)$ forms a base for $\left(\mathcal{B}^{X}, \mu_{\Gamma}\right)$;

(iv) Every b-uniform net space is a b-uniform ring space [17];

(v) Every bounded b-uniform filter space $\left(X, \mathcal{B}^{X}, \mu\right)$ is a b-uniform ring space, where $\left(\mathcal{B}^{X},\left\{B \times B: B \in \mathcal{B}^{X}\right\}\right)$ forms a base for $\left(\mathcal{B}^{X}, \mu\right)$;

(vi) For a reordered set-convergence space $\left(X, \mathcal{B}^{X}, q\right)$ the space $\left(X, \mathcal{B}^{X}, \mu_{q}\right)$ defines a b-uniform ring space, where the pair $\left(\mathcal{B}^{X},\{\dot{B} \times \mathcal{F}: \mathcal{F} q B, B \in\right.$ $\left.\left.\mathcal{B}^{X}\right\}\right)$ forms a base for $\left(\mathcal{B}^{X}, \mu_{q}\right)$;

(vii) For a neighbourhood space $\left(X, \mathcal{B}^{X}, \Theta\right)$, [23] the space $\left(X, \mathcal{B}^{X}, \mu_{\Theta}\right)$ defines a b-uniform ring space, where $\left(\mathcal{B}^{X},\left\{\dot{B} \times \Theta(B): B \in \mathcal{B}^{X}\right\}\right)$ forms a base for $\left(\mathcal{B}^{X}, \mu_{\Theta}\right)$.

Proposition 4.6. A pair $\left(\mathcal{A}^{X}, \eta\right)$ is a base for a b-uniform ring structure iff it satisfies the conditions in 4.2. and in addition the following one, i.e.

(rbas) $\mathcal{U} \in \eta$ implies the existence of $\mathcal{V} \in \eta$ s.t. $\mathcal{V} \subset \mathcal{U} \circ \mathcal{U}$. 
Remark 4.7. Here we again assume that all mentioned bases in 4.5 even define the condition (rbas). So let us call a pair $\left(\mathcal{A}^{X}, \eta\right)$ satisfying these conditions a ring base. Now in applying this new definition we characterize pre-Chy filters in a buniform ring space $\left(X, \mathcal{B}^{X}, \mu\right)$ by a given ring base $\left(\mathcal{A}^{X}, \eta\right)$ as follows:

$\mathcal{C} \in F I L(X)$ is a pre-Chy filter in $\left(\mathcal{B}^{X}, \mu\right)$ iff $\exists \mathcal{U} \in \eta \forall R \in \mathcal{U} \exists A \in \mathcal{A}^{X}$ s.t. $R(A) \in \mathcal{C}$.

In 4.5 (iv) we pointed out that b-uniform net spaces are in fact b-uniform ring spaces. Now, for generated b-uniform filter spaces the following statements are equivalent:

(i) $\left(X, \mathcal{B}^{X}, \mu\right)$ is a b-uniform net space;

(ii) $\left(X, \mathcal{B}^{X}, \mu\right)$ is a b-uniform ring space.

Proof. Evident.

Remark 4.8. In this context we also mention that for a generated b-uniform filter space $\left(X, \mathcal{B}^{X}, \mu\right),\left(X, \mathcal{B}^{X}, \mu\right)$ is a b-uniform net space iff $\left(X, \mathcal{B}^{X}, \cap \mu\right)$ defines a quasi-uniform space, provided that $\mathcal{B}^{X}$ is discrete with $\cap \mu:=\cap\{\mathcal{U}: \mathcal{U} \in \mu\}$.

Note that $(X, \mathcal{U})$ is a quasi-uniform space iff the principal preuniform convergence space $(X,[\mathcal{U}])$ defines a quasiuniform convergence space [1]. At the end of this section we add the fact that each b-uniform filter space $\left(X, \mathcal{B}^{X}, \mu\right)$ induces a b-uniform ring space $\left(X, \mathcal{B}^{X}, \stackrel{\circ}{\mu}\right)$ by defining a ring base $\left(\mathcal{B}^{X}, \stackrel{\circ}{b}\right)$ with $\stackrel{\circ}{b}:=\{\mathcal{U} \in \mu: \mathcal{U} \subset \mathcal{U} \circ \mathcal{U}\}$. The identity map $1_{X}:\left(X, \mathcal{B}^{X}, \stackrel{\circ}{\mu}\right) \longrightarrow\left(X, \mathcal{B}^{X}, \mu\right)$ is b-uniform continuous by applying 4.3 , and moreover if $\left(Y, \mathcal{B}^{Y}, \mu_{Y}\right)$ is b-uniform ring space and $f:\left(Y, \mathcal{B}^{Y}, \mu_{Y}\right) \longrightarrow\left(X, \mathcal{B}^{X}, \mu\right)$ an injective b-uniformly continuous map, then $f:\left(Y, \mathcal{B}^{Y}, \mu_{Y}\right) \longrightarrow\left(X, \mathcal{B}^{X}, \dot{\mu}\right)$ is b-uniformly continuous, too.

Thus, each b-uniform filter space $\left(X, \mathcal{B}^{X}, \mu\right)$ has a restricted co-universal map with respect to the inclusion functor $F: \mathbf{b}$-URING $\longrightarrow$ b-UFIL, where bURING denotes the full subcategory of b-UFIL, whose objects are the b-uniform ring spaces.

Theorem 4.9. b-URING forms a topological construct [22].

Proof. For any set $X$, the class $\left\{\left(Y, \mathcal{B}^{Y}, \mu\right) \in \mid \mathbf{b}\right.$-URING $\left.\mid: X=Y\right\}$ of all b-URING objects with underlying set $X$ is a set, because of $\left(\mathcal{B}^{Y}, \mu\right) \in \underline{P}(\underline{P} X) \times$ $\underline{P}(F I L(X \times X))$.

The only b-uniform ring structure on a set $X$ with $\operatorname{Card} X=1$ is the pair $(\{\emptyset,\{x\},\{\dot{x} \times \dot{x}, \underline{P}(\{x\} \times\{x\})\})$, where $x$ denotes the element of $X$. If $X$ is empty, then $(\{\emptyset\},\{\{\emptyset\}\})$ represents the only b-uniform ring structure on $X$.

For a set $X$, let $I$ be a class, $\left(X_{i}, \mathcal{B}^{X_{i}}, \mu_{i}\right)_{i \in I}$ a family of b-uniform ring spaces and $\left(f_{i}: X \longrightarrow X_{i}\right)_{i \in I}$ a family of maps. Then, $\left(\mathcal{B}_{I}^{X}, \mu_{X}^{I}\right)$ is the initial b-URING structure on $X$, where

$$
\begin{aligned}
& \mathcal{B}_{I}^{X}:=\left\{B \subset X: \forall i \in I f_{i}[B] \in \mathcal{B}^{X_{i}}\right\} \text { and } \\
& \mu_{I}^{X}:=\left\{\mathcal{U} \in F I L(X \times X): \forall i \in I\left(f_{i} \times f_{i}\right)(\mathcal{U}) \in \mu_{i}\right\} .
\end{aligned}
$$

Then, the remaining is clear. 
Remark 4.10. The initial b-uniform ring structure on a set $X$ with respect to $\left.\left(X, f_{i},\left(X_{i}, \mathcal{B}^{X_{i}}, \mu_{i}\right), I\right)\right)$ is the coarsest b-uniform ring structure on $X$ such that $f_{i}$ is b-uniformly continuous for each $i \in I$.

Specifically, let $\left(X, \mathcal{B}^{X}, \mu\right)$ be a b-uniform ring space and $A \subset X$. Then, $\left(\mathcal{B}^{A}, \mu_{A}\right)$ is b-uniform ring structure on $A$, where

$$
\begin{aligned}
& \mathcal{B}^{A}:=\left\{B \cap A: B \in \mathcal{B}^{X}\right\} \text { and } \\
& \mu_{A}:=\left\{\mathcal{U}_{A}: \mathcal{U} \in \mu\right\} \text { with } \mathcal{U}_{A}:=\{R \cap(A \times A): R \in \mathcal{U}\},
\end{aligned}
$$

such that $\left(A, \mathcal{B}^{A}, \mu_{A}\right)$ represents the b-uniform ring subspace of $\left(X, \mathcal{B}^{X}, \mu\right)$ in $\mathbf{b}$ URING.

\section{The Ultracompletion of A B-Uniform Ring SPACE}

Now, in answering the main question of this paper, we will construct an ultracompletion for an arbitrary non-empty b-uniform ring space and then apply this result to some former treated special constructs. In addition we study certain separation properties especially those the space may possess to carry over to the ultracompletion. Here, we extend an idea for quasi-uniform spaces due to Carlson and Hicks [3] as indicated in the following:

Construction 5.1. Let $\left(X, \mathcal{B}^{X}, \mu\right)$ be a non-empty b-uniform ring space. Then, we put $X^{*}:=X \cup\{\infty\}$ with $\infty \notin X$. For $\mathcal{U} \in \mu$ we are setting:

$$
\mathcal{U}^{*}:=\left\{R^{*} \subset X^{*} \times X^{*}: \exists R \in \mathcal{U} R^{*} \supset R \cup\left\{(\infty, x): x \in X^{*}\right\}\right\} \text { and }
$$

$\mathcal{B}^{X^{*}}:=\mathcal{B}^{X} \cup\{\{\infty\}\}$. Then, $\left(\mathcal{B}^{X^{*}}, \underline{b}^{*}\right)$ forms a base for a b-uniform ring structure $\left(\mathcal{B}^{X^{*}}, \mu^{*}\right)$ on $X^{*}$, where $\underline{b}^{*}:=\left\{\mathcal{U}^{*}: \mathcal{U} \in \mu\right\}$, compare with 4.7.

Proof. Evidently $\mathcal{B}^{X^{*}}$ defines a B-set on $X^{*}$. Also note that $\mathcal{U}^{*} \in F I L\left(X^{*} \times X^{*}\right)$ holds. As next we infer that for $\mathcal{U} \in \mu$

$\{$ i $\left.\}\left(R \cup\{\infty, x): x \in X^{*}\right\}\right)(\{\infty\})=X^{*}$ for each $R \in \mathcal{U}$ and

\{ii $\left.\}\left(R \cup\{\infty, x): x \in X^{*}\right\}\right)(B)=R(B)$ for every $R \in \mathcal{U}$ and each $B \in \mathcal{B}^{X} \backslash\{\emptyset\}$ are valid.

$\left(X^{*}, \mathcal{B}^{X^{*}}, \mu^{*}\right)$ is ultracomplete, because the following holds:

Let $\mathcal{C}^{*}$ be a pre-Chy filter in $\left(\mathcal{B}^{X^{*}}, \mu^{*}\right)$. We will show that the statement $\mathcal{C}^{*} q_{\mu^{*}}\{\infty\}$ can be deduced, meaning that $\dot{\infty} \times \mathcal{C}^{*} \in \mu^{*}$ is true. Therefore, it suffices to verify that the inclusion $\dot{\infty} \times \mathcal{C}^{*} \supset \mathcal{U}^{*}$ holds for some $\mathcal{U}^{*} \in \underline{b}^{*}$.

By the hypothesis and according to 4.7 we can find $\mathcal{V}^{*} \in \underline{b}^{*}$ with the corresponding property. Since $\underline{b}^{*}$ is a ring base we can find $\mathcal{U}^{*} \in \underline{b}^{*}$ with $\mathcal{U}^{*} \subset \mathcal{V}^{*} \circ \mathcal{V}^{*}$. Our goal is to verify that $\mathcal{U}^{*} \subset \dot{\infty} \times \mathcal{C}^{*}$ holds.

$R^{*} \in \mathcal{U}^{*}$ implies $R^{*} \supset V^{*} \circ V^{*}$ for some $V^{*} \in \mathcal{V}^{*}$, where $V^{*} \supset V \cup\{(\infty, x)$ : $\left.x \in X^{*}\right\}$ for some $V \in \mathcal{V}$. By applying the corresponding property for $\mathcal{C}^{*}$ we can find $D \in \mathcal{B}^{X^{*}}$ with $V^{*}(D) \in \mathcal{C}^{*}$. We claim that $\{\infty\} \times V^{*}(D) \subset V^{*} \circ V^{*}$ is true. But $(y, z) \in\{\infty\} \times V^{*}(D)$ implies the existence of $x^{\prime} \in D$ such that $\left(x^{\prime}, z\right) \in V^{*}$ with $y=\infty$.

Since $V \cup\left\{(\infty, x): x \in X^{*}\right\} \subset R^{*}$ we obtain $\left(y, x^{\prime}\right) \in V^{*}$. Thus $(y, z) \in$ $V^{*} \circ V^{*} \subset R^{*}$ follows, concluding the proof. 
Next we infer that the inclusion map $i:\left(X, \mathcal{B}^{X}, \mu\right) \longrightarrow\left(X^{*}, \mathcal{B}^{X^{*}}, \mu^{*}\right)$ is buniformly continuous, and $\left(X, \mathcal{B}^{X}, \mu\right)$ is b-uniform ring subspace of $\left(X^{*}, \mathcal{B}^{X^{*}}, \mu^{*}\right)$, compare with 4.10 . But this can be done in a straightforward manner. Finally, we have to verify that $X$ is dense in $X^{*}$, which means that the equation $c^{\mu^{*}}(X)=X^{*}$ can be deduced, compare with 3.6. So let $z \in X^{*}$ and without restriction $z=\infty$.

Choose $\mathcal{U} \in \mu$, hence $\mathcal{U}^{*} \in \underline{b}^{*}$ follows. It remains to verify that $\{z\} \times X \in \sec \mathcal{U}^{*}$ holds. For $R^{*} \in \mathcal{U}^{*}$ we can find $R \in \mathcal{U}$ s.t. $R^{*} \supset\left\{(\infty, x): x \in X^{*}\right\} \cup R$. By choosing $x \in X$ we obtain $(z, x) \in\{z\} \times\left(X \cap R^{*}\right)$, and the claim immediately follows.

Definition 5.2. Let $\left(X, \mathcal{B}^{X}, \mu\right)$ be a non-empty b-uniform ring space and $\left(X^{*}, \mathcal{B}^{X^{*}}, \mu^{*}\right)$ the ultracomplete b-uniform ring space as constructed in 5.1 , then the pair $\left(i,\left(X^{*}, \mathcal{B}^{X^{*}}, \mu^{*}\right)\right)$ is called the ultracompletion of $\left(X, \mathcal{B}^{X}, \mu\right)$ (sometimes only the space $\left(X^{*}, \mathcal{B}^{X^{*}}, \mu^{*}\right)$ will be called as above stated).

Separation properties come into play if one is considering convergence in a more suitable sense. This is also of importance if universal properties are examined. In the next we are giving some fundamental definitions in this direction.

Definition 5.3. A set-convergence $\left(\mathcal{B}^{X}, q\right)$ is called

(i) $T_{0}$ set- convergence, and the triple $\left(X, \mathcal{B}^{X}, q\right) T_{0}$ set-convergence space iff it satisfies the following condition, i.e.

$\left(\mathrm{T}_{0}\right) B_{1}, B_{2} \in \mathcal{B}^{X} \backslash\{\emptyset\}, \dot{B_{1}} q B_{2}$ and $\dot{B_{2}} q B_{1}$ imply $B_{1}=B_{2}$;

(ii) $T_{1}$ set-convergence, and the triple $\left(X, \mathcal{B}^{X}, q\right) T_{1}$ set-convergence space iff it satisfies the following condition, i.e.

$\left(\mathrm{T}_{1}\right) B_{1}, B_{2} \in \mathcal{B}^{X} \backslash\{\emptyset\}$ and $\dot{B}_{1} q B_{2}$ imply $B_{1}=B_{2}$;

(iii) $T_{2}$ set-convergence, and the triple $\left(X, \mathcal{B}^{X}, q\right) T_{2}$ set-convergence space iff it satisfies the following condition, i.e.

$\left.\mathrm{T}_{2}\right) \mathcal{F} \in F I L(X), B_{1}, B_{2} \in \mathcal{B}^{X} \backslash\{\emptyset\}$ with $\mathcal{F} q B_{1}$ and $\mathcal{F} q B_{2}$ imply $B_{1}=B_{2}$.

Consequently, we call a b-uniform filter space $\left(X, \mathcal{B}^{X}, \mu\right) T_{0}$ space $\left(T_{1}\right.$ space, $T_{2}$ space, respectively) iff $\left(X, \mathcal{B}^{X}, q_{\mu}\right)$ is a $T_{0}$ set- convergence space $\left(T_{1}\right.$ setconvergence space, $T_{2}$ set-convergence space, respectively). Then, related to the latter constructs we also speak of a $T_{0}$ ring space $\left(T_{1}\right.$ ring space, $T_{2}$ ring space, respectively), and these should be also done when considering b-uniform net spaces.

Remark 5.4. Now, it can be easily seen that $T_{2}$ implies $T_{1}$ and $T_{1}$ implies $T_{0}$. On the other hand we point out that in the discrete case these definitions coincide with those occurring in the theory of point-convergence spaces in the sense of Preuss [22].

Lemma 5.5. For a reordered set-convergence space $\left(X, \mathcal{B}^{X}, q\right)$ each successive pair of conditions are equivalent:

$\{\mathrm{i}\}\left(\mathcal{B}^{X}, q\right)$ is $T_{0}$ set-convergence;

$\{$ ii $\} \quad x, z \in X, \dot{x} q\{z\}$ and $\stackrel{\bullet}{z} q\{x\}$ imply $x=z$;

$\{$ iii $\}\left(\mathcal{B}^{X}, q\right)$ is $T_{1}$ set-convergence;

$\{$ iv $\} \quad x, z \in X$ and $\dot{x} q\{z\}$ imply $x=z$; 
$\{\mathrm{v}\}\left(\mathcal{B}^{X}, q\right)$ is $T_{2}$ set-convergence;

$\{$ vi $\} \mathcal{F} \in F I L(X), x, z \in X$ and $\mathcal{F} q\{x\}, \mathcal{F} q\{z\}$ imply $x=z$.

Proof. Evident.

Proposition 5.6. For a $T_{0}$ ring space $\left(X, \mathcal{B}^{X}, \mu\right)$ the completion $\left(X^{*}, \mathcal{B}^{X^{*}}, \mu^{*}\right)$ is an $T_{0}$ ring space.

Proof. Straightforward.

Remark 5.7. At this point we note that $\left(X^{*}, \mathcal{B}^{*}, \mu^{*}\right)$ is never $T_{1}$ space, and thus it is also not $T_{2}$ space. Now, the following question naturally arises. Does a $T_{2}$ ring space or $T_{1}$ ring space have an $T_{2}$ ultracompletion or $T_{1}$ ultracompletion, respectively? But this problem is not the aim of our present paper.

Proposition 5.8. Let $\left(X, \mathcal{B}^{X}, \mu\right)$ be a non-empty generated b-uniform ring space and $\left(X^{*}, \mathcal{B}^{*}, \mu^{*}\right)$ its ultracompletion. Then, the ultracompletion is generated, too.

Proof. Note that the following inclusion $(\cap \mu)^{*} \subset \cap \mu^{*}$ holds.

Remark 5.9. Taking 4.8 into account we point out that in the discrete case the associated quasi-uniform space of the ultracompletion is up to isomorphism the one- point completion of a given quasi-uniform space in the sense of Carlson and Hicks [3], and thus it is even strongly complete in their terminology. In this context we mention that for a discrete b-uniform filter space $\left(X, \mathcal{B}^{X}, \mu\right)$ and for every $\mathcal{F} \in F I L(X)$ the following statements are equivalent:

(i) $\mathcal{F}$ is pre-Chy filter in $\left(\mathcal{B}^{X}, \mu\right)$;

(ii) $\exists \mathcal{U} \in \mu \forall R \in \mathcal{U} \exists x \in X$ with $R(\{x\}) \in \mathcal{F}$.

By taking 5.8 into account, $\mathcal{F} \in F I L(X)$ is pre-Chy filter in a generated b-uniform filter structure $\left(\mathcal{B}^{X}, \mu\right)$ iff $\forall R \in \cap \mu, \exists B \in \mathcal{B}^{X} \backslash\{\emptyset\}$ s.t. $R(B) \in \mathcal{F}$.

Thus if combining both statements in the discrete case we obtain the usual classical property of a filter for being Cauchy.

Theorem 5.10. For a generated final b-uniform filter space $\left(X, \mathcal{B}^{X}, \mu\right)$ the following statements are, equivalent:

(i) $\left(X, \mathcal{B}^{X}, \mu\right)$ is ultrabounded;

(ii) $\forall R \in \cap \mu \exists B \subset X$ finite $R(B)=X$.

Proof. to (ii) $\Rightarrow$ (i): Let $\mathcal{F} \in F I L(X) \backslash\{\underline{P} X\}$ be an ultrafilter and $R \in \cap \mu$. Then, we can find points $x_{1}, \ldots, x_{n} \in X$ such that $X=R\left(x_{1}\right) \cup \ldots \cup R\left(x_{n}\right)$ by applying the hypothesis.

But $\mathcal{F}$ is ultrafilter, and thus $R\left(x_{i}\right) \in \mathcal{F}$ for some $x_{i}$. Consequently, $\mathcal{F}$ is pre-Chy filter in $\left(\mathcal{B}^{X}, \mu\right)$.

to (i) $\Rightarrow$ (ii): If $(i)$ is not true, then we can find a relation $R \in \cap \mu$ such that $R(B) \neq X$ for all $B \subset X$ finite.

The set $\{X \backslash R(B): B \subset X$ finite $\}$ forms a base for a filter $\mathcal{F} \in F I L(X) \backslash\{\underline{P} X\}$. Let $\mathcal{C}$ be an ultrafilter containing $\mathcal{F}$. Then, by the hypothesis $\mathcal{C}$ is pre-Chy filter in $\left(\mathcal{B}^{X}, \mu\right)$. Hence we can find $B_{1} \in \mathcal{B}^{X} \backslash\{\emptyset\}$ with $R\left(B_{1}\right) \in \mathcal{C}$. But this is a contradiction because by the hypothesis $B_{1}$ is finite, and thus the claim follows. 
Theorem 5.11. For a non-empty generated final ultrabounded b-uniform ring space $\left(X, \mathcal{B}^{X}, \mu\right)$ its ultracompletion $\left(X^{*}, \mathcal{B}^{X^{*}}, \mu^{*}\right)$ is final ultrabounded, too.

Proof. By the construction and hypothesis $\left(X^{*}, \mathcal{B}^{X^{*}}, \mu^{*}\right)$ is final. In addition we have that $\cap \mu \in \mu$ is valid, hence $(\cap \mu)^{*} \in \underline{b}^{*}$ follows. So let $R^{*} \in(\cap \mu)^{*}$, then $R^{*} \supset R \cup\left\{(\infty, x): x \in X^{*}\right\}$ for some $R \in \cap \mu$. By applying 5.10 we can find $B \subset X$ finite with $R(B)=X$. We put $B^{*}:=B \cup\{\infty\}$, hence $B^{*} \subset X^{*}$ is finite. So it remains to verify that the equation $R^{*}\left(B^{*}\right)=X^{*}$ holds.

Let $z \in X^{*}$, in the case of $z \in X$, we can choose $x \in B$ with $(x, z) \in R$. Consequently, $x \in B^{*}$ and $(x, z) \in R^{*}$ follow, showing that $z \in R^{*}\left(B^{*}\right)$ is true. In the other case, $z=\infty$ implies $z \in B^{*}$, and $(z, z) \in R^{*}$ follows, showing the claim, too.

Theorem 5.12. For a non-empty generated final ultrabounded b-uniform ring space $\left(X, \mathcal{B}^{X}, \mu\right)$ the b-uniform ring space $\left(X^{*}, \mathcal{B}^{X^{*}}, \mu^{*}\right)$ is ultracompact.

Proof. By applying 2.9 and the former obtained results.

Remark 5.13. The outcome just obtained may be of importance, if one intends to consider generalized proximities defined in terms of final ultrabounded b-uniform ring structures. But this line of vision may be left to the reader.

Applied Resume 5.14. At the end of this section we still mention the facts that each non-empty b-uniform net space as well as every non-empty merotopically b-uniform filter space has an ultracompletion, too [17]. Consequently, as a corollary we obtain the result that each quasiuniform limit space has an ultracompletion if supposing the discrete case. Secondly, we can state, that a non-empty merotopically b-uniform filter space possesses at least two different completions, namely the one mentioned above and, in addition, the Cauchy-completion, dealt with in $[17]$.

\section{SOME IMPORTANT LINKS TO GENERALIZED APPROACH SPACES}

The central idea in approach spaces in the sense of Lowen is that of a distance $d$, which is a function on $X \times 2^{X}$ to $[0, \infty]$. Here of fundamental interest is the fact that a distance can be defined not only in a metric space, but also in a topological space, a uniform space and so on. This setting is in fact well motivated by Lowens original axioms for an approach space in terms of its point-set function $d: X \times 2^{X} \longrightarrow$ $[0, \infty]$ listed in $[18,21]$. Now, we get some interesting links to our former presented concepts of b-uniform filter spaces, set-convergence spaces, b-topological spaces, see 6.4 or bornological spaces, respectively, see also [10].

First we will give the definition of a so-called approach-bornology on a set $X$.

Definition 6.1. For a set $X$ a pair $\left(\mathcal{B}^{X}, d\right)$ consisting of non-empty subset $\mathcal{B}^{X} \subset \underline{P} X$ and a distance function $d: X \times \mathcal{B}^{X} \longrightarrow[0, \infty]$ is called an approach-bornology, shortly apbornology and the triple $\left(X, \mathcal{B}^{X}, d\right)$ approach-bornological space (shortly apbornological space or apb space, respectively), provided that the following conditions are satisfied,

(apb $\left.b_{1}\right) B_{1} \subset B \in \mathcal{B}^{X}$ imply $B_{1} \in \mathcal{B}^{X}$;

$\left(\right.$ apb $\left._{2}\right) x \in X$ implies $\{x\} \in \mathcal{B}^{X}$; 
$\left(\mathrm{apb}_{3}\right) B_{1}, B_{2} \in \mathcal{B}^{X}$ imply $B_{1} \cup B_{2} \in \mathcal{B}^{X}$

$\left(\mathrm{apb}_{4}\right) x \in X$ implies $d(x, \emptyset)=\infty$;

$\left(\right.$ apb $\left._{5}\right) x \in X$ implies $d(x,\{x\})=0$;

$\left(\right.$ apb $\left._{6}\right) x \in X$ and $B_{1}, B_{2} \in \mathcal{B}^{X}$ imply $d\left(x, B_{1} \cup B_{2}\right)=\min \left\{d\left(x, B_{1}\right), d\left(x, B_{2}\right)\right\}$.

If $\left(X, \mathcal{B}^{X}, d_{X}\right),\left(Y, \mathcal{B}^{Y}, d^{Y}\right)$ are apb spaces then a function $f: X \longrightarrow Y$ is called $b$-contracted map provided it satisfies following conditions,

$\left(\right.$ bct $\left._{1}\right) f$ is bounded;

(bct bor $_{2}$ foach $x \in X$ and for each $B \in \mathcal{B}^{X}$ the unequality $d_{Y}(f(x), f[B]) \leq$ $d_{X}(x, B)$ holds.

By APB we are denoting the category of apb spaces and b-contracted maps.

Remark 6.2. Here, we point out that $\mathcal{B}^{X}$ defines a bornology on $X$ in the sense of Hogbe-Nlend [9]. In addition we note that the operator $c l_{d}: \mathcal{B}^{X} \longrightarrow \underline{P X}$ defined by setting $c l_{d}(B):=\{x \in X: d(x, B)=0\}$ forms a b-closure operator on $\mathcal{B}^{X}$ provided that $c l_{d}(B) \in \mathcal{B}^{X}$ is valid for every $B \in \mathcal{B}^{X}[14]$.

In this context we can specify the term b-contracted by adding that $f$ is also rebounded, if necessary, which means for each $D \in \mathcal{B}^{Y}, f^{-1}[D] \in \mathcal{B}^{X}$ is valid. We also point out that in the case of $\mathcal{B}^{X}=\underline{P} X$, the corresponding space is called pre-approach space by F. Mynard and E. Pearl in their book Beyond Topology [20]. Here one can find additional propositions concerning this construct.

Furthermore, motivated by Lowen's idea to define a distance in a topological space we will generalize this concept to a b-closure operator(b-closure) as indicated in the following:

Definition 6.3. In accordance with Leseberg [14], we call a pair $\left(\mathcal{B}^{X}, h\right)$, where $\mathcal{B}^{X}$ is bornology and $h: \mathcal{B}^{X} \longrightarrow \underline{P} X$ a function, called $b$-closure operator, a $b$ closure structure $\left(\right.$ on $\left.\mathcal{B}^{X}\right)$ and the triple $\left(X, \mathcal{B}^{X}, h\right)$ b-closure space, provided that the following conditions are satisfied:

$\left(\right.$ bclo $\left._{1}\right) B \in \mathcal{B}^{X}$ implies $h(B) \in \mathcal{B}^{X}$;

$\left(\mathrm{bclo}_{2}\right) h(\emptyset)=\emptyset$;

(bclo $\left._{3}\right) x \in X$ implies $x \in h(\{x\})$;

(bclo $\left.)_{4}\right) B_{1} \subset B \in \mathcal{B}^{X}$ imply $h\left(B_{1}\right) \subset h(B)$;

$\left(\right.$ bclo $\left._{5}\right) B_{1}, B_{2} \in \mathcal{B}^{X}$ imply $h\left(B_{1} \cup B_{2}\right) \subset h\left(B_{1}\right) \cup h\left(B_{2}\right)$.

If $\left(X, \mathcal{B}^{X}, h^{X}\right),\left(Y, \mathcal{B}^{Y}, h^{Y}\right)$ are b-closure spaces, then a function $f: X \longrightarrow Y$ is called $b$-continuous map provided $f$ satisfies the following conditions:

$\left(\mathrm{bc}_{1}\right) f$ is bounded;

$\left(\mathrm{bc}_{2}\right) f$ is rebounded;

$\left(\mathrm{bc}_{3}\right) B \in \mathcal{B}^{X}$ implies $f\left[h^{X}(B)\right] \subset h^{Y}(f[B])$.

By b-CLO we are denoting the category of b-closure spaces and b-continuous maps.

Remark 6.4. As an important supplement we should note that a b-closure structure $\left(\mathcal{B}^{X}, h\right)$ is called b-topology and the triple $\left(X, \mathcal{B}^{X}, h\right)$ b-topological space provided that, in addition, the following condition holds:

(btop) $B \in \mathcal{B}^{X}$ implies $h(h(B)) \subset h(B)$.

Then, $h$ is called b-topological operator. By b-TOP we denote the full subcategory of b-CLO whose objects are the b-topological spaces [14]. 
Now, we will give an intrinsic example for the former introduced concept as follows:

Example 6.5. Let a bornological space $\left(X, \mathcal{B}^{X}\right)$ be given and $x \in X$ be a point of $X$. Then, we define a b-topological operator $t^{x}: \mathcal{B}^{X} \longrightarrow \underline{P} X$ with fix-point $x$ by setting:

$$
\begin{array}{ll}
t^{x}(\emptyset):=\emptyset & \text { and } \\
t^{x}(B):=\{x\} \cup B & \text { for } B \in \mathcal{B}^{X} \backslash\{\emptyset\} .
\end{array}
$$

Remark 6.6. With the now introduced concept it is possible to consider topologies not only on the power set $\underline{P} X$ of $X$, but also on subsets such as the set of all finite sets, compact sets or totally bounded sets, respectively. In the case of $\mathcal{B}^{X}$ being saturated, meaning that $X \in \mathcal{B}^{X}$ holds, b-topological spaces and topological spaces are essentially the same (up to isomorphism). On the other hand classical closure spaces are extensively examined in the book of Čech, so that the concept of b-closure operators also makes sense.

Proposition 6.7. In a b-closure space $\left(X, \mathcal{B}^{X}, h\right)$ we set $\delta_{h}(x, B):=0$ iff $x \in h(B)$ and $\delta_{h}(x, B):=\infty$, otherwise. Then, the pair $\left(\mathcal{B}^{X}, \delta_{h}\right)$ satisfies the conditions for an apbornology, and it is compatible with the b-closure operator $h$.

Proof. Evidently, $c l_{\delta_{h}}$ defines a b-closure operator, see also 6.2, and the following sequence of equivalences is valid, i.e. $x \in c l_{\delta_{h}}(B) \Leftrightarrow \delta_{h}(x, B)=0 \Leftrightarrow x \in$ $h(B)$.

Remark 6.8. As seen above to make sure that in general $c l_{d}: \mathcal{B}^{X} \longrightarrow \underline{P} X$ forms a b-closure operator it is necessary to provide the condition in 6.2 . Now the question arises, whether the associated b-closure $\left(\mathcal{B}^{X}, c l_{d}\right)$ is compatible with the given apbornology $\left(\mathcal{B}^{X}, d\right)$. To this end, we are giving the following condition:

Definition 6.9. An apbornology $\left(\mathcal{B}^{X}, d\right)$ is called covered, and the triple $\left(X, \mathcal{B}^{X}, d\right)$ covered apbornological space (shortly covered apb space) provided that the following conditions are satisfied:

$\left(\operatorname{cov}_{1}\right) B \in \mathcal{B}^{X}$ implies $c l_{d}(B) \in \mathcal{B}^{X}$

$\left(\operatorname{cov}_{2}\right) B \in \mathcal{B}^{X}$ and $x \notin c l_{d}(B)$ imply $d(x, B)=\infty$.

By COV-APB we denote the subcategory of APB, whose objects are covered.

Proposition 6.10. For a covered apbornological space $\left(X, \mathcal{B}^{X}, d\right)$ the following equation holds, i.e. $\delta_{c l_{d}}=d$.

Proof. Let $x \in X$ and $B \in \mathcal{B}^{X}$. In the case of $x \in c l_{d}(B)$, we have $d(x, B)=0$ and $\delta_{c l_{d}}(x, B)=0$, which shows the equality. If $x \notin c l_{\delta}(B)$, then $d(x, B)=\infty$ by applying $\left(\operatorname{cov}_{2}\right)$. But on the other hand $\delta_{c l_{d}}(x, B)=\infty$ also follows, thus implying the claim. So it remains to verify that $\delta_{h}$ is satisfying $\left(\operatorname{cov}_{2}\right)$, because by 6.3 we have $c l_{\delta_{h}}(B)=h(B) \in \mathcal{B}^{X}$, thus implying $\left(\operatorname{cov}_{1}\right)$. $x \notin c l_{\delta_{h}}(B)$ implies $x \notin h(B)$, hence $\delta_{h}(x, B)=\infty$, so that $\delta_{h}$ fulfills $\left(\operatorname{cov}_{2}\right)$.

Proposition 6.11. For b-closure spaces $\left(X, \mathcal{B}^{X}, h^{X}\right),\left(Y, \mathcal{B}^{Y}, h^{Y}\right)$ let $f: X \longrightarrow$ $Y$ be a function, then the following statements are equivalent:

(i) $f:\left(X, \mathcal{B}^{X}, h^{X}\right) \longrightarrow\left(Y, \mathcal{B}^{Y}, h^{Y}\right)$ is b-continuous;

(ii) $f:\left(X, \mathcal{B}^{X}, \delta_{h^{X}}\right) \longrightarrow\left(Y, \mathcal{B}^{Y}, \delta_{h^{Y}}\right)$ is b-contracted. 
Proof. Consider remark 6.2.

to (i) $\Rightarrow$ (ii): So let $x \in X$ and $B \in \mathcal{B}^{X}$. In the case of $x \in h^{X}(B), \delta_{h^{X}}(x, B)=$ 0 , and by the hypothesis $f(x) \in h^{Y}(f[B])$ follows, showing that $\delta_{h^{Y}}(f(x), f[B])=$ 0 is true, and the claim in (ii) results. In the case of $x \notin h^{X}(B), \delta_{h^{X}}(x, B)=\infty$ is valid. Since $\delta_{h^{Y}}(f(x), f[B]) \in[0, \infty]$ holds we obtain $\delta_{h^{Y}}(f(x), f[B]) \leq \infty=$ $\delta_{h^{x}}(x, B)$, and the claim follows.

to (ii) $\Rightarrow(\mathrm{i})$ : Let $B \in \mathcal{B}^{X}$ and $y \in f\left[h^{X}(B)\right]$. Our goal is that $\delta_{h^{Y}}(y, f[B])=0$ can be deduced.

We have $y=f(x)$ for some $x \in h^{X}(B)$, and consequently $\delta_{h^{x}}(x, B)=0$ follows. But by the hypothesis $f$ is b-contracted, i.e. $\delta_{h^{Y}}(f(x), f[B]) \leq \delta_{h^{x}}(x, B)$ holds, and the claim results.

Theorem 6.12. The categories $\boldsymbol{C O} \boldsymbol{V}-\boldsymbol{A P B}$ and $\boldsymbol{b}-\boldsymbol{C L} \boldsymbol{O}$ are isomorphic.

Proof. Evident, by applying 6.4, 6.5, 6.6, 6.7, 6.8, 6.9 and 6.10, respectively.

To obtain a corresponding embedding of $\mathbf{b}-\mathbf{T O P}$ into APB we have to specify the concept of a covered apbornology as follows:

Definition 6.13. A covered apbornology $\left(\mathcal{B}^{X}, d\right)$ is called topoform, and the triple $\left(X, \mathcal{B}^{X}, d\right)$ topoform apb space, provided that the following condition is satisfied,

(top) $x \in X$ and $B \in \mathcal{B}^{X}$ imply $d(x, B) \leq d\left(x, c l_{d}(B)\right)$.

By TOP-APB we denote the full subcategory of CLO-APB, whose objects are topoform.

Remark 6.14. It is easy to see that if $\left(\mathcal{B}^{X}, d\right)$ forms a topoform apbornology, then $c l_{d}: \mathcal{B}^{X} \longrightarrow \underline{P} X$ defines a b-topological operator on $\mathcal{B}^{X}$.

Theorem 6.15. The categories TOP-APB and b-TOP are isomorphic.

Proof. By applying the results obtained earlier and taking into account the following last reflection, i.e. for a b-topological operator $t: \mathcal{B}^{X} \longrightarrow \underline{P} X, x \in$ $X$ and $B \in \mathcal{B}^{X}$ we consider the two distances, i.e. $\delta_{t}(x, B)$ and $\delta_{t}\left(x, c l_{\delta_{t}}(B)\right)$, respectively. In the case of $x \in t(B), \delta_{t}(x, B)=0$. On the other hand we get $x \in t(t(B))$, hence $\delta_{t}(x, t(B))=0$ implying $\left.x \in c l_{\delta_{t}}(t(B))=c l_{\delta_{t}}(B)\right)$, thus $\delta_{t}\left(x, c l_{\delta_{t}}(B)\right)=0$, and the claim follows. If secondly $x \notin t(B)$, then $\delta_{t}(x, B)=$ $\infty$ is valid. On the other hand we have $x \notin t(t(B))$, since $t$ satisfies (btop). Consequently, $\infty=\delta_{t}(x, t(B))=\delta_{t}\left(x, c l_{\delta_{t}}(B)\right)$ follows, showing the claim too.

Remark 6.16. Our last result now makes sure that the category APB and the category of generalized near spaces, denoted by SD [17] have a non-empty intersection, which contains b-TOP.

Next we will give a slight modification for the conditions of being an apbornology.

Definition 6.17. For a bornology $\mathcal{B}^{X}$ let $d: X \times \mathcal{B}^{X} \longrightarrow[0, \infty]$ be a function so that $\left(\mathcal{B}^{X}, d\right)$ satisfies the following conditions, i.e.

$($ pre-apb 1$) \quad x \in X$ implies $d(x, \emptyset)=\infty$;

$($ pre-apb $) \quad x \in B \in \mathcal{B}^{X}$ imply $d(x, B)=0$. 
Then, $\left(\mathcal{B}^{X}, d\right)$ is called a pre-apbornology (on $\left.X\right)$ and the triple $\left(X, \mathcal{B}^{X}, d\right)$ preapbornological space (shortly pre-apb space). By PRE-APB we denote the category, whose objects are the pre-apb spaces and whose morphisms between them satisfy the conditions in 6.1 .

Remark 6.18. Evidently, every apbornological space is a pre-apb space.

Now, let us return to the concept of pointset- convergence spaces, compare also with [19].

Proposition 6.19. For a pointset- convergence space $\left(X, \mathcal{B}^{X}, q\right)$ (see also 3.2), we put:

$$
\begin{aligned}
& d_{q}(x, B)=0 \quad \text { iff } \dot{x} \quad q B \text { and } \\
& d_{q}(x, B)=\infty \quad \text { otherwise. }
\end{aligned}
$$

Then, $\left(\mathcal{B}^{X}, d_{q}\right)$ forms a pre-apbornology.

Proof. to $($ pre-apb 1$) d_{q}(x, \emptyset)=\infty$ holds, since $\dot{x}$ is not in relation with $\emptyset$. Otherwise $\dot{x}=\underline{P} X$ is a contradiction.

to (pre-apb 2 ) For $x \in B \in \mathcal{B}^{X} \quad \dot{B} \quad q B$ and $\dot{B} \subset \dot{x}$ are valid, implying $\dot{x} \quad q B$, hence $d_{q}(x, B)=0$ follows.

Conversely, for a pre-apbornology $\left(\mathcal{B}^{X}, \delta\right)$ we are setting:

$$
\begin{array}{lll}
\mathcal{F} p_{\delta} \emptyset & \text { iff } & \mathcal{F}=\underline{P} X \text { and } \\
\mathcal{F} p_{\delta} B & \text { iff } & \forall x \in B \exists F \in \mathcal{F} \cap \mathcal{B}^{X} \delta(x, F)=0 \text { for } B \in \mathcal{B}^{X} \backslash\{\emptyset\} .
\end{array}
$$

Then, obviously $\left(\mathcal{B}^{X}, p_{\delta}\right)$ defines a pointset-convergence.

In this context we mention that convergence on approach spaces is extensively studied by Lowen or Brock $[2,19]$, respectively under name of convergence approach spaces. So it was shown that the axiom (F) defined for limit spaces by Cook and Fischer can be extended to an axiom (F) for convergence approach spaces so that the full subcategory of CAP (convergence approach spaces), whose objects satisfy $(\mathrm{F})$ is isomorphic to the category $\mathbf{A P}$ of approach spaces.

Now, the question arises, whether $\left(\mathcal{B}^{X}, d_{q}\right)$ is compatible with the pointsetconvergence $\left(\mathcal{B}^{X}, q\right)$, meaning that $p_{\delta_{q}}=q$ can be deduced. But this seems only to be possible by adding suitable properties for the given pointset-convergence as done in the following:

Definition 6.20. A pointset-convergence $\left(\mathcal{B}^{X}, q\right)$ is called rotary and the triple $\left(X, \mathcal{B}^{X}, q\right)$ rotary pointset-convergence space, provided that the following conditions are satisfied, i.e. For $\mathcal{F} \in F I L(X)$ and each $x \in X$ let being valid:

$$
\begin{aligned}
& \left(\text { rot }_{1}\right) F \in \mathcal{F} \cap \mathcal{B}^{X} \text { and } \dot{x} q F \text { imply } \mathcal{F} q\{x\} ; \\
& \left(\operatorname{rot}_{2}\right) \mathcal{F} q\{x\} \text { implies } \exists F \in \mathcal{F} \cap \mathcal{B}^{X} \text { s.t. } \dot{x} q F .
\end{aligned}
$$

By RP-SETCONV we denote the full subcategory of P-SETCONV, whose objects are rotary.

Proposition 6.21. Let a rotary pointset-convergence space $\left(X, \mathcal{B}^{X}, q\right)$ be given, then the pre-apbornology $\left(\mathcal{B}^{X}, d_{q}\right)$ as defined in 6.19 is compatible. 
Proof. So let without restriction $B \in \mathcal{B}^{X} \backslash\{\emptyset\}$ and at first $\mathcal{F} p_{d_{q}} B$. Then, for each $x \in B$ we can find $F \in \mathcal{F} \cap \mathcal{B}^{X}$ such that $d_{q}(x, F)=0$ is valid. Hence $\dot{x} q F$ follows, and $\mathcal{F} q\{x\}$ results by applying ( $\left.\operatorname{rot}_{1}\right)$. Consequently, we obtain $\mathcal{F} q B$, since by the hypothesis $\left(\mathcal{B}^{X}, q\right)$ is a pointset-convergence.

Conversely, for $\mathcal{F} q B$ let $x \in B$. Since $\left(\mathcal{B}^{X}, q\right)$ is reordered $\mathcal{F} q\{x\}$ follows implying the existence of $F \in \mathcal{F} \cap \mathcal{B}^{X}$ with $\stackrel{\bullet}{x} \quad q F$ by applying ( $\operatorname{rot}_{2}$ ), hence $d_{q}(x, F)=0$, and $\mathcal{F} p_{d_{q}} B$ results.

To ensure that the pointset-convergence $\left(\mathcal{B}^{X}, p_{\delta}\right)$ is rotary the pre-apbornology $\left(\mathcal{B}^{X}, \delta\right)$ has to be reflexive in the following sense:

Definition 6.22. A pre-apbornology $\left(\mathcal{B}^{X}, d\right)$ is called reflexive and the triple $\left(X, \mathcal{B}^{X}, d\right)$ an reflexive pre- apb space provided that the following condition holds:

(ref) $z \in X, F \in \mathcal{B}^{X}$ and $d(z, F)=0$ imply $x=z$ for every $x \in F$.

By RPRE-APB we denote the full subcategory of PRE-APB, whose objects are reflexive.

Remark 6.23. For a pre-apbornology $\left(\mathcal{B}^{X}, d\right)$ let $d$ be injective, then $\left(\mathcal{B}^{X}, d\right)$ is reflexive. On the other hand for a reflexive apbornology $\left(\mathcal{B}^{X}, d\right)$ the bounded sets of $\mathcal{B}^{X}$ are closed meaning that $c l_{d}(B)=B$ holds for every $B \in \mathcal{B}^{X}$.

Proposition 6.24. For a reflexive pre-apbornology $\left(\mathcal{B}^{X}, \delta\right)$ the pointset-convergence $\left(\mathcal{B}^{X}, p_{\delta}\right)$ is a rotary $T_{1}$ set-convergence.

Proof. to $\left(\operatorname{rot}_{1}\right):$ For $\mathcal{F} \in F I L(X), x \in X$ and $F \in \mathcal{F} \cap \mathcal{B}^{X}$ let $\dot{x} p_{\delta} F$. Choose $z \in F$, hence by the hypothesis we can find $F_{1} \in \dot{x} \cap \mathcal{B}^{X}$ with $\delta\left(x, F_{1}\right)=0$. But $x \in F_{1}$ implies $z=x$, because $\left(\mathcal{B}^{X}, d\right)$ is reflexive. Consequently, $x \in F$ follows, and according to $($ pre-apb 2$) \delta(x, F)=0$ results implying $\mathcal{F} p_{\delta}\{x\}$.

to $\left(\operatorname{rot}_{2}\right): \mathcal{F} p_{\delta}\{x\}$ implies the existence of $F \in \mathcal{F} \cap \mathcal{B}^{X}$ with $\delta(x, F)=0$. Let $z \in F$, hence $x=z$ by the hypothesis, and $x \in F$ follows, showing that $F \in \dot{x}$ is true. But $\left(\mathcal{B}^{X}, p_{\delta}\right)$ is a pointset-convergence, and the claim results.

to $\left(T_{1}\right)$ : For $x, z \in X$ let $\dot{x} p_{\delta}\{z\}$, hence we can find $F \in \dot{x} \cap \mathcal{B}^{X}$ with $\delta(z, F)=0$ implying $x \in F$, and by the hypothesis $x=z$ follows (compare also with 5.5).

Proposition 6.25. For a $T_{1}$ pointset-convergence $\left(\mathcal{B}^{X}, q\right),\left(\mathcal{B}^{X}, d_{q}\right)$ is reflexive.

Proof. $z \in X, F \in \mathcal{B}^{X}$ and $d_{q}(x, F)=0$ imply $\dot{x} \quad q F$ by the definition. Let $x \in F$, then we get $\dot{x} q\{z\}$ according to (RO), and $x=z$ follows, since $\left(\mathcal{B}^{X}, q\right)$ is $T_{1}$ set-convergence.

Definition 6.26. A reflexive pre-apbornology $\left(\mathcal{B}^{X}, \delta\right)$ is called convergent, and the triple $\left(X, \mathcal{B}^{X}, \delta\right)$ convergent pre-apb space, provided that the following condition is satisfied:

(conv) $x \in X, B \in \mathcal{B}^{X}$ and $\dot{x}$ is not in relation under $p_{\delta}$ with $B$ imply $\delta(x, B)=\infty$. By CPRE-APB we denote the full subcategory of RPRE-APB, whose objects are convergent. 
Proposition 6.27. For a convergent pre-apbornology $\left(\mathcal{B}^{X}, \delta\right)$ the equation

$$
d_{p_{\delta}}=\delta
$$

holds.

Proof. Let $x \in X$ and $B \in \mathcal{B}^{X}$. In the first case of $\dot{x} p_{\delta} B$, we have $d_{p_{\delta}}(x, B)=$ 0 . On the other hand choose $z \in B$, hence $\delta(z, B)=0$. Moreover by the definition of $p_{\delta}$ we can find $F \in \dot{x} \cap \mathcal{B}^{X}$ with $\delta(z, F)=0$. But $x \in F$ implies $x=z$, since $\left(\mathcal{B}^{X}, \delta\right)$ is reflexive, and $\delta(x, B)=0$ follows which shows the claim. Secondly, if $\dot{x}$ is not in relation under $p_{\delta}$ with $B$ then $d_{p_{\delta}}(x, B)=\infty=\delta(x, B)$ are true by applying the hypothesis.

Now if we denote by $\mathbf{T}_{\mathbf{1}} \mathbf{R P}$-SETCONV the full subcategory of

\section{RP-SETCONV}

whose objects are $T_{1}$ set- convergence spaces we obtain the following result:

Theorem 6.28. The categories $\mathbf{T}_{\mathbf{1}} \mathbf{R P}-\mathbf{S E T C O N V}$ and $\mathbf{C P R E - A P B}$ are isomorphic.

Proof. Evident, by taking into account the last verified equations. So it only remains to prove the following equivalence of statements, i.e., for convergent preapb spaces $\left(X, \mathcal{B}^{X}, d^{X}\right),\left(Y, \mathcal{B}^{Y}, d^{Y}\right)$ let $f: X \longrightarrow Y$ be a map. Then, following statements are equivalent,

(i) $f:\left(X, \mathcal{B}^{X}, d^{X}\right) \longrightarrow\left(Y, \mathcal{B}^{Y}, d^{Y}\right)$ is b-contracted;

(ii) $f:\left(X, \mathcal{B}^{X}, p_{d^{X}}\right) \longrightarrow\left(Y, \mathcal{B}^{Y}, p_{d^{Y}}\right)$ is b-continuous.

to $(\mathrm{i}) \Rightarrow\left(\right.$ ii): Let $\mathcal{F} p_{d^{X}} \quad B$ and $y \in f[B]$, hence $y=f(x)$ for some $x \in B$. By the hypothesis there exists $F \in \mathcal{F} \cap \mathcal{B}^{X}$ with $d_{X}(x, F)=0$. Since $f$ is bcontracted $d^{Y}(f(x), f[F]) \leq d^{X}(x, F)=0$ and $f[F] \in f(\mathcal{F}) \cap \mathcal{B}^{Y}$ follow, implying $d^{Y}(y, f[F])=0$, and the claim results.

to (ii) $\Rightarrow$ (i): For $x \in X$ and $B \in \mathcal{B}^{X}$ we consider $d^{X}(x, B)$ as well as

$$
d^{Y}(f(x), f[B]) \text {. }
$$

But $d^{X}(x, B)=d_{p_{d} X}(x, B)$ and $d^{Y}(f(x), f[B])=d_{p_{d^{Y}}}(f(x), f[B])$.

In the case of $\dot{x} p_{d^{X}} B$, we have $d^{X}(x, B)=d_{p_{d} X}(x, B)=0$ and $\dot{f(x)} p_{d^{Y}} f[B]$. But $d_{Y}(f(x), f[B])=d_{p_{d}}(f(x), f[B])=0$ implies $d^{Y}(f(x), f[B]) \leq d^{X}(x, B)$. Secondly if $\stackrel{\bullet}{x}$ is not in relation under $p_{d^{x}}$ with $B$ we get $d^{X}(x, B)=d_{p_{d^{X}}}(x, B)=\infty$ with $d^{Y}(f(x), f[B]) \leq \infty$, and the claim follows.

Our next definition provides a closed connection between bornological spaces and apbornological spaces in the sence that BORN can be regarded as a full embedded subcategory of APB. Hence in view of 1 and 4.5, respectively b-URING and APB intersects.

Definition 6.29. An apbornology $(\mathcal{B}, \delta)$ is called elementary, and the triple $\left(X, \mathcal{B}^{X}, \delta\right)$ an elementary apbornological space provided that the following condition is satisfied,

(e) $x \notin B$ implies $\delta(x, B)=\infty$. 
By E-APB we denote the full subcategory of APB, whose objects are elementary.

Theorem 6.30. The categories E-APB and BORN are isomorphic.

Proof. For a bornological space $\left(X, \mathcal{B}^{X}\right)$ we define a distance function $d_{b}^{X}$ : $X \times \mathcal{B}^{X} \longrightarrow[0, \infty]$ by setting for each pair $(x, B) \in X \times \mathcal{B}^{X}$ :

$$
\begin{array}{ll}
d_{b}^{X}(x, B):=0 & \text { iff } x \in B \\
d_{b}^{X}(x, B):=\infty, & \text { otherwise. }
\end{array}
$$

Then, the natural corresponding assignments are functorial and determine the announced isomorphism between the two categories in question.

Remark 6.31. By applying 2.7 we can state that every non-empty elementary apbornological space is ultracomplete, up to isomorphism.

\section{REFERENCES}

[1] A. Behling, Einbettung uniformer Räume in topologische Universen, Dissertation, Freie Universität Berlin, Berlin, 1991.

[2] P. Brock, D. Kent, On convergence approach spaces, Applied Categorical Structures 6 (1998), 117-125.

[3] J. W. Carlson and T. L. Hicks, On completeness in quasi-uniform spaces, Journal of Mathematical Analysis and Applications 34 (1971), 618-627.

[4] Á. Császár, Foundation of General Topology, Pergamon Press, NY, 1963.

[5] D. Doitchinov, A concept of completeness of quasi-uniform spaces, Topology and its Applications 38 (1991), 205-217.

[6] S. Dolecki and F. Mynard, Convergence Foundation of Topology, World Scientific, 2016.

[7] P. Fletcher and W.F. Lindgren, Quasi-Uniform Spaces, Lecture Notes in Pure and Applied Mathematics 77, Marcel Dekker, NY, 1982.

[8] F. Hausdorff, Gestufte Räume, Fundamenta Mathematicae 25 (1935), 486-502.

[9] H. Hogbe-Nlend, Bornologies and Functional Analysis, North-Holland Mathematics Studies 26, North-Holland, Amsterdam, 1977.

[10] G. Jäger, Convergence approach spaces and approach spaces as lattice-valued convergence spaces, Iranian Journal of Fuzzy Systems 9 (2012), 1-16.

[11] M. Katětov, On continuity structures and spaces of mappings, Commentationes Mathematicae Universitatis Carolinae 6 (1965), 275-278.

[12] H.-P. A. Künzi, An introduction to quasi-uniform spaces, in: F. Mynard and E. Pearl (eds.), Beyond Topology, Contemporary Mathematics 486, American Mathematical Society, 2009, 239-304.

[13] A. Lechicki, S. Levi and A. Spakowski, Bornological convergences, Journal of Mathematical Analysis and Applications 297 (2004), 751-770.

[14] D. Leseberg, Extensions in bounded topology, Mathematics for Application 3 (2014), 97-113.

[15] D. Leseberg and Z. Vaziry, Categories of several convergences, Submitted to Journal of the American Mathematical Society.

[16] D. Leseberg and Z. Vaziry, The quasitopos of b-uniform filter spaces, Mathematics for Applications 7 (2018), 155-171.

[17] D. Leseberg and Z. Vaziry, The Cauchy completion of a symmetric b-uniform filter space, Submitted to Journal of Applied Analysis.

[18] R. Lowen, Approach Spaces: The Missing Link in the Topology-Uniformity-Metric-Triad, Oxford Mathematical Monographs, Oxford University Press, 1997.

[19] E. Lowen and R. Lowen, A quasitopos containing CONV and MET as full subcategory, International Journal of Mathematics and Mathematical Sciences 11 (1988), 417-438.

[20] F. Mynard and E. Pearl (eds), Beyond Topology, Contemporary Mathematics 486, American Mathematical Society, 2009. 
[21] S. A. Naimpally, Featured Review MR1472024 (98f:54002) of the Book [18], American Mathematical Society, 1998.

[22] G. Preuss, Foundation of Topology: An Approach to Convenient Topology, Springer, 2002.

[23] A. Tozzi and O. Wyler, On categories of supertopological spaces, Acta Universitatis Carolinae. Mathematica et Physica 28 (1987), 137-149.

[24] T. Vroegrijk, On the semi-uniformity of bornological convergence, Quaestiones Mathematicae 38 (2015), 285-296.

[25] O. Wyler, On convergence of filters and ultrafilters to subsets, in: H. Ehrig, H. Herrlich, H.-J. Kreowski and G. Preuss (eds.), Categorical Methods in Computer Science With Aspects from Topology, Lecture Notes in Computer Science 393, 1988, 340-350.

Dieter Leseberg, Ernst Reuter Gesellschaft Berlin, Germany e-mail: leseberg@zedat.fu-berlin.de

Zohreh Vaziry, Department of Mathematics, Karaj Branch, Islamic Azad University, Karaj, Iran

e-mail: $z_{-} \mathrm{m}_{-}$vaziry@yahoo.co.in 
